\title{
Problema de estoque e roteirização: revisão bibliográfica
}

\author{
Patrícia Prado Belfiore \\ Oswaldo Luiz do Valle Costa \\ POLI-USP \\ Luiz Paulo Lopes Fávero \\ FEA-USP
}

\section{Resumo}

Este trabalho apresenta os principais modelos encontrados na literatura do problema de estoque e roteirização, tanto com demanda determinística quanto estocástica. 0 problema consiste em determinar quando e quanto entregar de mercadoria para cada cliente e quais roteiros de entregas utilizar, com o objetivo de minimizar os custos de estoque e distribuição, de modo que as demandas de todos os clientes sejam atendidas.

\section{Palavras-chave}

Problema de estoque e roteirização, estoque gerenciado pelo fornecedor, pesquisa operacional.

\section{Inventory routing problem: bibliographic review}

\begin{abstract} minimize inventory and distribution costs, guaranteeing that all the clients' demands are met.

Key words

Inventory routing problem, VMI (Vendor Managed Inventory), operational research.
\end{abstract}

This paper presents the main models found in the literature of inventory routing problem, with both deterministic and stochastic demand. The problem consists of determining when and how much merchandise to deliver, aiming to 


\section{INTRODUĈ̣̃O}

Nas últimas décadas, tendo em vista o aumento da competição global e os avanços tecnológicos, a logística passou a exercer um papel fundamental na gestão dos negócios empresariais. Nesse contexto, a satisfação do cliente torna-se um elemento fundamental no mercado atual e engloba disponibilidade do produto, agilidade e eficiência na entrega, entre outros elementos, fazendo com que as empresas busquem meios de melhorar e reduzir os custos dos processos logísticos.

A eficiência e competitividade de cada empresa dependem do desempenho da cadeia de suprimentos, fazendo com que o ganho individual esteja diretamente inter-relacionado com o ganho total da cadeia (KLINGENBERG e ANTUNES, 2002).

Iniciativas importantes visando elevar os ganhos totais da cadeia têm surgido nas indústrias, com o amparo do Movimento ECR (Efficient Consumer Response) ou Resposta Eficiente ao Consumidor. O ECR consiste em uma série de princípios e estratégias que visam a introdução eficiente de novos produtos, a promoção eficiente, ao sortimento eficiente e a reposição eficiente de mercadorias (KLINGENBERG e ANTUNES, 2002).

Uma das técnicas propostas pelo ECR é o VMI (Vendor Managed Inventory) ou Estoque Gerenciado pelo Fornecedor, que tem sido muito disseminada na indústria mundial. O VMI tem como objetivo a redução de custos através da integração dos componentes da cadeia de suprimentos. O processo de reposição através do VMI pode ocorrer em qualquer elo da cadeia de suprimentos. Segundo Campbell et al. (1998), o VMI é uma das recentes tendências da logística.

Campbell et al. (1998) e Campbell, Clarke e Savelsbergh (2002) definem o VMI como uma técnica na qual o fornecedor controla os níveis de estoque de seus clientes e decide quando e quanto entregar de mercadoria para cada cliente. Sendo assim, os cálculos são realizados por um algoritmo cadastrado no fornecedor, formado por parâmetros preestabelecidos pelo vendedor e comprador e baseado nas informações obtidas do cliente. Nesse modelo, o cliente é apenas informado da quantidade que será enviada. Desta maneira, ele faz um acompanhamento, monitora, mas não controla o processo.

$\mathrm{O}$ primeiro passo para o sucesso da técnica VMI requer disponibilidade e acurácia dos dados dos clientes. Para tomar decisões, o fornecedor tem acesso a informações importantes, como: níveis de estoques (disponibilidade de produtos) atual e passado de todos os clientes, comportamento da demanda do consumidor para previsão da demanda futura, capacidade de estocagem dos clientes, distância e tempo de viagem dos clientes em relação ao fornecedor e entre eles, custos de transporte, custos de manutenção de estoques, cus- to de falta de estoque e capacidade e disponibilidade de veículos e motoristas para entrega dos produtos (KLEYWEGT, NORI e SAVELSBERGH, 2004).

Como exemplos de indústrias que utilizam o VMI, têmse: indústria petroquímica (transporte de gás), indústria de varejo (supermercados), indústria de bebidas (vendedoras de máquinas), e indústria automobilística (distribuição de autopeças). O número de indústrias utilizando a técnica VMI está crescendo rapidamente, em função dos avanços tecnológicos, através de modernos equipamentos que permitem ao vendedor medir os níveis de estoques dos clientes e comunicar-se com o seu sistema através do uso de scanners, computadores e modems (CAMPBELL, CLARKE e SAVELSBERGH, 2002).

\section{A eficiência e competitividade de cada empresa dependem do desempenho adeia de suprimentos.} estoques dos clientes, também administra uma frota de veículos para transportar os produtos aos clientes. Neste caso, o objetivo do vendedor é não só administrar o reabastecimento ótimo dos estoques, como também a distribuição dos produtos. Este problema é chamado problema de estoque e roteirização (Inventory Routing Problem - IRP). O IRP tem como característica a técnica VMI e desenvolve metodologias para solução deste problema. Através da modelagem e otimização da cadeia de suprimentos, a empresa pode melhorar o seu desempenho operacional e continuar competitiva. O IRP é resultado das novas formas de modelagem e otimização da cadeia de suprimentos baseadas na idéia de integração dos diversos componentes logísticos (KLEYWEGT, NORI e SAVELSBERGH, 2002, 2004).

Nos problemas tradicionais, os clientes controlam seus próprios níveis de estoques, e quando um cliente acha que é hora de reabastecer, faz o pedido de uma quantidade de produto para o fornecedor em função de suas necessidades. $\mathrm{O}$ fornecedor recebe os pedidos dos clientes e faz as entregas utilizando uma frota de veículos. Este problema é conhecido como problema de roteirização de veículos (CAMPBELL, CLARKE e SAVELSBERGH, 2002).

No problema de roteirização de veículos tradicional o horizonte de tempo é de curto prazo, normalmente de um único dia. O objetivo é determinar rotas de veículos que minimizem os custos de transporte, de modo que as demandas de todos os clientes sejam atendidas, e as restrições de capacidade dos veículos sejam respeitadas (CAMPBELL, CLARKE e SAVELSBERGH, 2002). 
Já no IRP o problema é complexo, o horizonte de planejamento é maior. O objetivo é determinar a cada dia quais clientes visitar e quanto entregar para cada um deles, de forma a minimizar os custos de estoque e distribuição, sob a restrição de que as demandas dos clientes sejam atendidas (CAMPBELL, CLARKE e SAVELSBERGH, 2002).

De acordo com Kleywegt, Nori e Savelsbergh (2002, 2004), o VRP, como não tem como característica a técnica VMI, tem algumas desvantagens. Não há troca de informações, o fornecedor tem um lead-time alto e alta variabilidade no volume dos pedidos dos clientes atacadistas e varejistas. Já o cliente trabalha com estoques elevados e possui nível de serviço baixo com alguns itens do fornecedor. Como resultado, toda cadeia de suprimentos trabalha com um nível de estoques elevado. Há uma distorção na demanda. Os pedidos não chegam para o fornecedor uniformemente ao longo do tempo. Como resultado, os recursos do fornecedor, tais como produção, estoques, recursos de transporte, não são utilizados da melhor forma possível. Durante um período chegam vários pedidos, enquanto que no resto do período há ociosidade. Outra desvantagem é que, como o fornecedor não tem o controle do nível de estoque do cliente, quando recebe pedidos urgentes, não consegue diferenciar a prioridade entre os pedidos, ou seja, qual pedido é realmente urgente.

Ainda segundo Kleywegt, Nori e Savelsbergh (2002, 2004), o problema de estoque e roteirização, que tem como característica a técnica VMI, beneficia tanto o vendedor quanto o cliente. Do lado do fornecedor, primeiramente, podem-se reduzir os custos de produção e estoque. A utilização de recursos é mais uniforme, o que reduz o montante dos recursos necessários, aumenta a produtividade dos mesmos, e reduz o nível de estoque. Em segundo lugar, podem-se reduzir os custos de transporte através de uma utilização mais uniforme da capacidade de transporte. Fazer o planejamento através de uma informação disponível ao invés de basear-se em pedidos de clientes resulta num planejamento mais eficiente. Para o cliente, as vantagens são: o aumento do nível de serviço, em termos de disponibilidade do produto, e o fato de que ele investe menos recursos no controle do nível de estoque e pedidos.

Se a técnica VMI beneficia tanto o fornecedor quanto os clientes, e é relativamente barata, por que não é aplicada em grande escala? A razão é, com certeza, a difícil tarefa de desenvolver uma estratégia de distribuição que minimize os custos totais de distribuição e estoque. Quanto maior o número de clientes envolvidos, mais difícil torna-se o problema. Por um lado, a flexibilidade de decidir quando e quanto entregar reduzirá significativamente os custos de distribuição e estoque. Por outro lado, esta flexibilidade dificulta a obtenção de uma boa solução e, mais ainda, da solução ótima de distribuição e estoque (CAMPBELL et al., 1998).
Este trabalho faz uma revisão dos principais modelos e métodos de resolução do IRP encontrados na literatura, com o objetivo de contribuir para a futura aplicação destas técnicas no contexto brasileiro.

\section{DEFINIC̣ÃO DO PROBLEMA DE ESTOQUE E ROTEIRIZAC̣ÃO}

O problema de estoque e roteirização (IRP) trata da distribuição de um ou mais produtos, a partir de um ou mais centros de distribuição, que atendem $N$ clientes dentro de um horizonte de planejamento $T$. Cada cliente $i$ consome o produto a uma taxa $v_{i}$ e tem uma capacidade de armazenagem $C_{i}$. O nível de estoque do cliente $i$ no instante $t$ é $I_{i}^{t}$. A distribuição dos produtos pode ser feita através de uma frota de veículos homogênea ou heterogênea, e cada veículo tem uma capacidade $C_{v}$. A quantidade entregue ao cliente $i$ no instante $t$ é $Q_{i}^{t}$. Define-se $c_{e s t, i}$ o custo de armazenagem do cliente $i$.

O objetivo é minimizar o custo médio diário de distribuição durante o horizonte de planejamento, de modo que não haja falta de estoques para os clientes. Podem-se adicionar ao modelo custos de estoque, custos de falta (admitindo que pode ocorrer falta de produtos) e até mesmo a função lucro em função dos produtos entregues ou das vendas.

A cada instante $t$, são tomadas decisões de roteamento de veículos e reabastecimento de estoque dos clientes. As decisões são tomadas diariamente.

O custo de uma decisão no instante $t$ pode incluir:

- Custo de transporte $c_{i j}$ dos $\operatorname{arcos}(i, j)$;

- Lucro: se for entregue uma quantidade $Q_{i}^{t}$ ao cliente $i$ no instante $t$, o vendedor tem um lucro de $L_{i}\left(Q_{i}^{t}\right)$;

- Penalidade de falta $p_{i}\left(s_{i}^{t}\right)$ se a demanda $s_{i}^{t}$ do cliente $i$ no dia $t$ não for atendida. A demanda não atendida é tratada como demanda perdida e não atraso na entrega;

- Custo de estoque $c_{\text {est }, i}$ que pode ser definido como $c_{\text {est }, i}$ $\left(I_{i}^{t-1}+Q_{i}^{t}-v_{i}\right)$, sendo que:

$I_{i}^{i}-1-$ nível de estoque do cliente $i$ no dia anterior.

$Q_{i}^{t}$ - quantidade entregue ao cliente $i$ no dia $t$.

$v_{i}$ - demanda diária do cliente $i$.

O custo de estoque também pode ser modelado como uma função da média de estoque de cada cliente durante o período de tempo.

O problema de estoque e roteirização engloba três decisões:

- Quando atender cada cliente;

- Quanto entregar de mercadoria para cada cliente;

- Qual o melhor roteiro de entrega. 


\section{CLASSIFICAC̣̃̃O DOS PROBLEMAS DE ESTOQUE E ROTEIRIZAC̣ÃO}

O IRP engloba vários elementos, portanto, a classificação deste problema não é uma tarefa simples. Diversas classificações para problemas de roteirização de veículos e modelos de estoque são encontradas na literatura, porém os mesmos não se aplicam diretamente a problemas de estoque e roteirização. Uma classificação para problemas de estoque e roteirização foi proposta por Baita et al. (1998) e está apresentada no Quadro 1. Esta classificação foi, posteriormente, apresentada por Znamenky e Cunha (2003).
Esta classificação não abrange todas as características, hipóteses e restrições de problemas de estoque e roteirização, pois se fossem consideradas todas as possibilidades, o número de combinações seria muito grande. Os autores destacam as principais classes, em função da importância e influência de suas características. Dentre as classes apresentadas no Quadro 1, destacam-se as categorias tipo de demanda e decisão.

O tipo de demanda pode ser classificado como determinística (conhecida) ou estocástica (desconhecida). Os problemas de estoque e roteirização são de natureza estocástica. Os modelos estocásticos, em geral, requerem grande

Quadro 1: Elementos de classificação para problemas de estoque e roteirização.

\begin{tabular}{|c|c|c|c|c|}
\hline Elemento & Atributo & \multicolumn{3}{|c|}{ Alternativas } \\
\hline $\begin{array}{c}\text { Topologia } \\
\text { da rede de } \\
\text { abastecimento }\end{array}$ & $\begin{array}{l}\text { Pontos de } \\
\text { abastecimento } \\
\text { ou distribuição }\end{array}$ & Um para um & Um para muitos & $\begin{array}{l}\text { Muitos } \\
\text { para } \\
\text { Muitos }\end{array}$ \\
\hline $\begin{array}{l}\text { Itens a serem } \\
\text { entregues }\end{array}$ & Número & Um & Muitos & \\
\hline \multirow{3}{*}{ Demanda } & Tipo & Determinística & Estocástica & \\
\hline & Comportamento & Constante & Variável & \\
\hline & $\begin{array}{c}\text { Distribuição } \\
\text { entre clientes }\end{array}$ & Uniforme & Não uniforme & \\
\hline Decisões & Domínio & $\begin{array}{l}\text { Freqüência de } \\
\text { atendimento }\end{array}$ & $\begin{array}{l}\text { Instante de } \\
\text { atendimento }\end{array}$ & \\
\hline \multirow{4}{*}{ Restrições } & $\begin{array}{l}\text { Capacidade } \\
\text { dos veículos }\end{array}$ & Sim (igual) & Sim (diferente) & Não \\
\hline & $\begin{array}{c}\text { Capacidade } \\
\text { de estocagem }\end{array}$ & Sim & Não & \\
\hline & $\begin{array}{c}\text { Capacidade } \\
\text { de abastecimento }\end{array}$ & Sim & Não & \\
\hline & $\begin{array}{l}\text { Número de } \\
\text { veículos }\end{array}$ & Fornecido & $\begin{array}{c}\text { Variável } \\
\text { de decisão }\end{array}$ & Não restritivo \\
\hline \multirow[b]{2}{*}{ Custos } & Estoque & Manutenção & Falta & Pedido \\
\hline & Distribuição & Fixo & $\begin{array}{l}\text { Proporcional à } \\
\text { distância }\end{array}$ & $\begin{array}{l}\text { Proporcional ao } \\
\text { número de clientes }\end{array}$ \\
\hline \multirow{4}{*}{$\begin{array}{l}\text { Estratégia } \\
\text { de solução }\end{array}$} & Decomposição & Tempo & Agrupa-roteiriza & \\
\hline & Agregação & Tempo & Freqüência & Distância \\
\hline & Algoritmo & Exato & Aproximado & \\
\hline & $\begin{array}{l}\text { Programação } \\
\text { matemática }\end{array}$ & Linear & Inteira & Não linear \\
\hline
\end{tabular}

Fonte: Adaptado de Baita et al. (1998) e Cunha (2003). 
quantidade de dados históricos e um tratamento estatístico adequado que nem sempre estão disponíveis. Por outro lado, os modelos com demanda determinística têm sido muito utilizados para resolução de problemas reais, introduzindo o estoque de segurança e incorporando a sazonalidade a um modelo de previsão de demanda, para absorver o comportamento estocástico (ZNAMENSKY e CUNHA, 2003).

Já a categoria decisão pode ser classificada como freqüência de atendimento ou instante de atendimento. No primeiro grupo, as decisões são tomadas em intervalos ou períodos regulares e o horizonte de planejamento é infinito. No segundo grupo, as variáveis de decisão são os instantes de entrega e o horizonte de planejamento é finito. Baita et al. (1998) dividem os modelos de freqüência de atendimento em três grupos:

1. Modelos agregados: correspondem aos modelos analíticos, que desenvolvem idéias qualitativas para sua solução. São, portanto, mais fáceis de serem resolvidos e comunicados, devido ao menor rigor matemático. Nesta categoria encontram-se os modelos que avaliam os tradeoffs entre os custos envolvidos no processo de decisão.

2. Modelos de política de partição fixa: nesta estratégia os clientes são, a priori, divididos em regiões de atendimento.
Cada vez que um veículo visita uma região de atendimento, todos os clientes deste grupo serão visitados.

3. Modelos de freqüiência discreta: ao invés de fracionários, os intervalos de atendimento são inteiros.

A Figura 1 apresenta essa classificação.

A seguir, são apresentados os principais métodos de solução encontrados na literatura para problemas de estoque e roteirização com demanda determinística e estocástica, de acordo com a classificação proposta na Figura 1. Posteriormente, cada um dos métodos apresentados é classificado de acordo com os elementos e atributos definidos no Quadro 1.

\section{MÉTODOS DE SOLUCÃO PARA PROBLEMAS DE ESTOQUE E ROTEIRIZAĈ̣̃O COM DEMANDA DETERMINÍSTICA}

\section{Modelos agregados (modelos de freqüência de atendimento]}

Blumenfeld et al. $(1985,1987)$ analisaram redes de distribuição com entrega direta de fornecedor para consumidor ou via terminais de consolidação. São analisados os trade-offs entre essas duas formas de distribuição e entre os custos de

Figura 1: Classificação de problemas de estoque e roteirização.

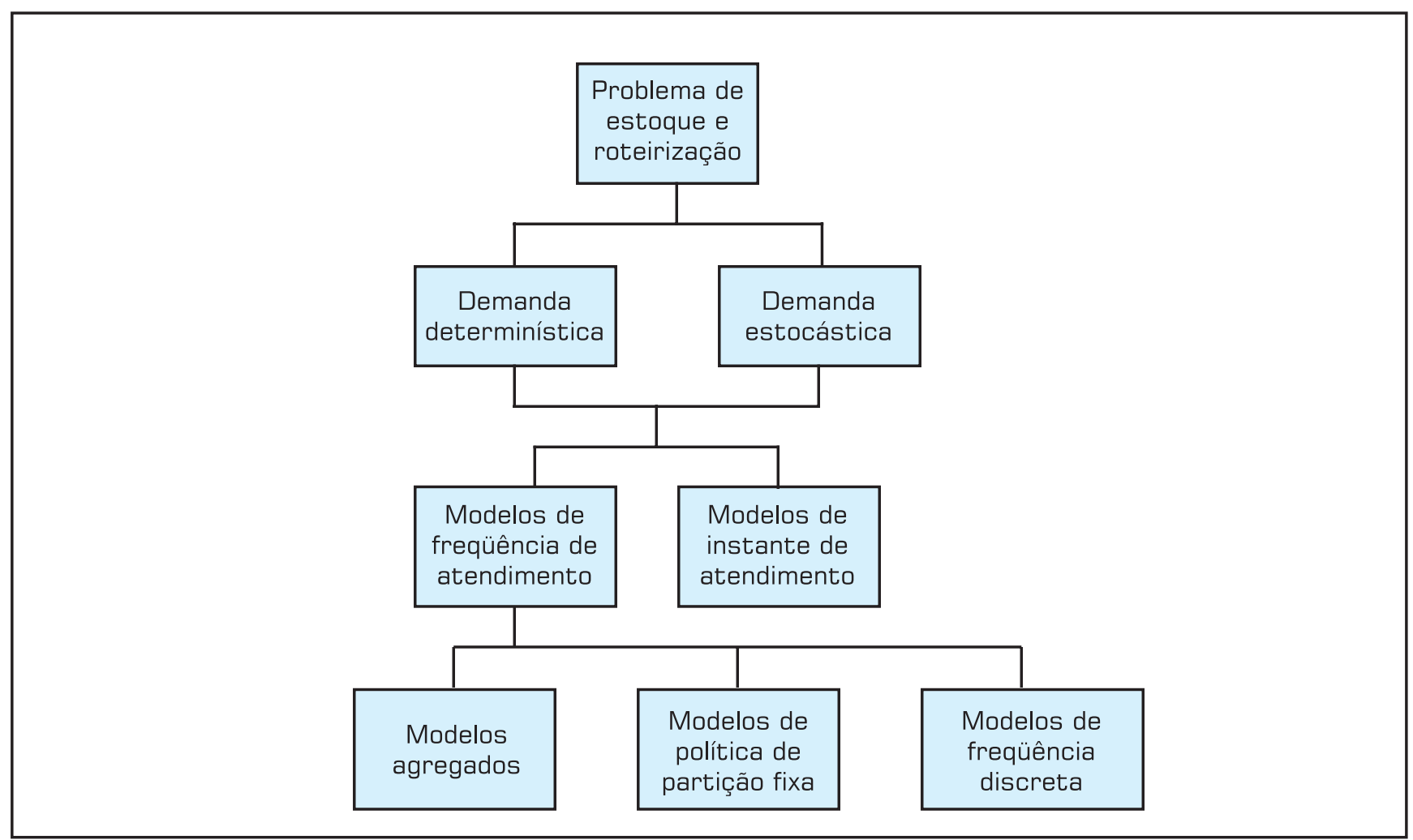

Fonte: Adaptado de Baita et al. (1998) e Cunha (2003). 
transporte, estoque e pedido. Os autores propõem um método simples de otimização, baseado no modelo do lote econômico de pedido (economic order quantity - EOQ). Uma extensão deste modelo é apresentada por Burns et al. (1985).

Burns et al. (1985) desenvolveram equações aproximadas aplicadas tanto no caso em que os veículos visitam apenas um cliente por rota quanto no caso de vários clientes por rota. O método analisa os trade-offs existentes entre os custos de transporte e estoque. Estes trade-offs são analisados tanto analiticamente, quanto geograficamente.

Larson (1988) propôs um método baseado na heurística das economias de Clarke e Wright (1964), que é aplicado com sucesso ao problema de coleta e transporte de resíduos gerados em estações de tratamento de água da cidade de Nova Iorque. O método considera rotas fixas para a coleta dos resíduos das estações de tratamento de esgoto, a consolidação em estações de transbordo, e o posterior transporte até seu destino final em alto-mar. É importante ressaltar que, neste caso, as demandas ou as quantidades de resíduos a serem coletados não são determinísticas, mas seguem uma distribuição normal, sendo, no entanto, tratadas como determinísticas a partir da especificação prévia de um nível de serviço. A utilização de rotas fixas faz com que alguns clientes tenham uma freqüência de visitas muito maior do que a necessária. Neste modelo todos os clientes de uma rota fixa são visitados no mesmo dia, mesmo os que não tenham necessidade de atendimento iminente.

Benjamin (1989) estudou o problema de distribuição de vários fornecedores para vários clientes. Partindo da decomposição do problema e da solução de maneira independente, das etapas de determinação do lote econômico de produção, do problema de transporte e da determinação do lote econômico de pedido, e considerando que a solução exata do problema envolve a resolução de um problema de programação não-linear, viável apenas para instâncias de menor porte, o autor apresenta uma heurística para a resolução simultânea dos três subproblemas de uma maneira conjunta. O modelo não considera roteiros de entrega, e sim apenas distribuição direta fornecedor-consumidor através de viagens redondas.

Uma pequena variação do problema de estoque e roteirização é a estratégia do problema de estoque e roteirização discutida por Webb e Larson (1995), que tem como objetivo minimizar o número de veículos para a entrega de mercadorias aos clientes a partir de um único depósito em problemas de estoque e roteirização. A informação é baseada na taxa de consumo dos clientes. O número mínimo de veículos deve ser capaz de continuar atendendo todos os clientes mesmo que haja uma variação na taxa de consumo dos clientes. Os clientes são divididos em grupos (clusters), e determina-se o roteiro para cada cluster. A partir daí, determina-se o nú- mero de veículos necessários. Os clientes podem pertencer a mais de uma rota. A seqüência de rotas é criada usando um modelo que minimiza a utilização do veículo, conseqüentemente minimiza o número de veículos. No modelo original de Larson (1988) todos os clientes de uma rota são visitados no mesmo dia, mesmo que não haja necessidade de atendimento iminente. Já no modelo de Webb e Larson (1995), os clientes são atendidos apenas quando necessário, resultando na otimização do custo da frota.

\section{Modelos de política de partição fixa (modelos de freqüência de atendimento)}

Anily e Federgruen (1990) desenvolveram uma heurística que minimiza os custos de estoque e transporte para um horizonte infinito, de forma que todos os clientes sejam atendidos. Assume-se que as demandas dos clientes são múltiplas de uma taxa base. Os custos de estoques são iguais para todos os clientes. Os custos de transporte incluem custos fixos (leasing ou aluguel) e custos variáveis proporcionais à distância. Os clientes são abastecidos a partir de um único depósito. Os estoques são mantidos nos clientes e não no depósito. Os clientes são divididos em regiões, de modo que a demanda de cada região seja igual à capacidade do veículo. Um cliente pode pertencer a mais de uma região. Quando o veículo visita um cliente na região, todos os clientes são visitados. Cada veículo é designado para uma região, e decide-se quanto entregar para cada cliente, a freqüência e a seqüência da rota. Este modelo adota, portanto, uma política de partição de clientes, definindo conjuntos fixos de clientes que serão tratados como regiões de atendimento.

Gallego e Simchi-Levi (1990) desenvolveram um modelo baseado nas idéias de Anily e Federgruen (1990), baseado na política de partição de clientes, para avaliar a eficácia de entregas diretas fornecedor-consumidor em um horizonte de longo prazo. O sistema de distribuição consiste de um único depósito e vários clientes dispersos geograficamente. Os estoques são mantidos apenas nos clientes, e não no depósito. Os autores apresentaram um limitante inferior com base nos custos de outras estratégias de estoque e roteirização, e concluíram que o modelo apresentado obteve melhores resultados em pelo menos $94 \%$ dos casos, na condição de que o tamanho do lote seja pelo menos $71 \%$ da capacidade do veículo. Portanto, o modelo não é viável quando a quantidade de mercadorias a ser entregue aos clientes for muito menor que a capacidade do veículo. 
A heurística de Anily e Federgruen (1990) foi criticada por Hall (1991), que mostrou que o modelo superestima os custos de distribuição quando do fracionamento da demanda dos clientes, uma vez que não se considera a possibilidade de coordenação entre as entregas ou compartilhamento dos estoques. Em resposta às críticas recebidas, Anily e Federgruen (1991) argumentam que tais deficiências são inerentes à política de abastecimento adotada, em que cada região da partição é tratada independente das demais, mesmo que alguns pontos de entrega de várias regiões correspondam a um mesmo cliente físico. Segundo os autores, a heurística não deve ser avaliada exclusivamente pelo desempenho do pior caso ou sua comparação com o mínimo custo possível, devendo ser considerados também aspectos positivos tais como a facilidade de implementação e administração da estratégia de solução proposta. que são servidos da mesma forma que as regiões de Anily e Federgruen (política de partição fixa).

Chan, Federgruen e Simchi-Levi (1998) analisam a política de estoque zero, na qual o nível de estoque do cliente é reabastecido somente quando não há estoque. Os autores também propõem uma heurística de partição de clientes baseada na resolução de um problema de localização de concentrador capacitado (Capacitated Concentrator Location Problem - CCLP), similar às idéias de Bramel e Simchi-Levi (1995).

\section{Modelos de freqüência discreta (modelos de freqüência de atendimento]}

Speranza e Ukovich (1994) estudaram o problema da distribuição de múltiplos produtos no caso de entregas diretas. O problema consiste em determinar as freqüências discretas das entregas de cada produto com o objetivo de minimizar os custos de transporte e estocagem. Os autores separam o problema segundo uma freqüência de atendimento adotada, única ou múltipla, e segundo o tipo de consolidação adotado por freqüência ou por instante de atendimento. O problema é modelado como programação linear inteira ou inteira mista.
O modelo de Anily e Federgruen (1993) trata da distribuição de um único produto, através de uma frota de veículos ilimitada e homogênea. O sistema de distribuição é feito a partir de um único depósito, que também serve como ponto de armazenagem, devendo incluir no modelo o custo de estoque e a capacidade do depósito, assim como o custo de reposição do estoque no depósito. Os autores propõem uma política de partição fixa similar a Anily e Federgruen (1990), porém nesse caso os intervalos entre atendimentos são arredondados para potências de dois. Este artifício permite que seja estimado o custo da política proposta em relação a resultados encontrados na literatura, o que segundo os autores não excede $6 \%$. O modelo considera custos de estoques iguais para todos os clientes e os custos de transporte incluem custos fixos (leasing ou aluguel) e custos variáveis proporcional à distância. $\mathrm{O}$ objetivo é minimizar os custos de estoque, transporte e pedidos num horizonte de longo prazo.

A partir das idéias de Gallego e Simchi-Levi (1990), Bramel e Simchi-Levi (1995) consideraram uma variação do problema de estoque e roteirização na qual os clientes podem ter um nível de estoques ilimitado. O problema foi transformado em um problema de localização do concentrador capacitado (CCLP - Capacitated Concentrator Location Problem). Para que o modelo tenha solução, resolve-se o CCLP e transforma-se a solução em uma solução para o IRP. A solução para o CCLP divide os clientes em grupos

\section{Modelos de instante de atendimento}

Bell et al. (1983) propuseram um modelo de programação inteira para o problema de roteirização e estoque da Air Products. As decisões são tomadas através de um sistema de decisão ou otimizador, que a partir de dados como taxa de consumo histórica dos clientes, tempos de viagem e distâncias, disponibilidade de motoristas, produtos e veículos, capacidade dos tanques e dos veículos, custos de transporte, define as rotas para os próximos 2 a 5 dias. O sistema utiliza um algoritmo de relaxação lagrangiana para resolver o modelo de programação inteira mista. Primeiramente, um programa define possíveis rotas com no máximo quatro clientes por rota a serem inclusas no modelo de programação inteira mista. Nem todas as possíveis rotas são selecionadas, e o programa utiliza uma heurística que decide se inclui ou não uma rota específica. As rotas que possuem um alto custo ou cuja demanda dos clientes difere consideravelmente da capacidade do veículo são descartadas. A ordem de visita precisa ser especificada. Definidas as possíveis rotas na primeira etapa, na segunda fase o modelo de programação mista decide, para cada instante de tempo, quais rotas serão selecionadas para cada veículo e a quantidade de mercadoria entregue para cada cliente no final do dia. O objetivo do modelo é maximizar a quantidade de produtos entregues e diminuir os custos de fazer estas entregas. Os custos considerados no modelo são: custos fixos, independentes da quantidade de mercadorias entregue, que 
variam conforme a distância, que são os custos do motorista, depreciação do veículo, combustível; e custo do veículo, que varia conforme a quantidade entregue. Os clientes são abastecidos a partir de um único depósito. Os veículos diferem em características como capacidade e custos de operação. As restrições do modelo são restrições de demanda e recursos (disponibilidade de veículos, motoristas e produto, capacidade do veículo). As restrições de demanda impõem limites inferiores e superiores na quantidade de produtos entregues.

Dror e Levy (1986) utilizaram um modelo de programação, para um horizonte de tempo de uma semana, aplicando trocas de nós e arcos para reduzir custos no horizonte de planejamento. O horizonte é reduzido de uma base anual para uma base semanal, ou seja, a solução para o problema de estoque e roteirização para uma base de tempo anual consiste de uma seqüência de soluções semanais consecutivas, que gera rotas diárias. Os clientes são reabastecidos por um depósito central, e o objetivo é minimizar o custo anual de distribuição de forma que não haja falta de estoque para nenhum cliente em nenhum momento. A partir de uma solução inicial, obtida através de uma adaptação do método de economias de Clarke e Wright (1964), são apresentadas três heurísticas de melhoria, que são capazes de examinar e operar todas as rotas simultaneamente, baseadas no conceito de trocas de nós em uma única rota ou entre várias rotas, similar a trocas de arcos em uma única rota. Este conceito de troca de nós ou troca de posições de clientes em uma rota ou entre rotas gera melhorias devido à flexibilidade de trocas de posições de dois nós de clientes, um nó, ou mesmo excluir ou inserir nós da solução de roteirização de veículos. Todos os três procedimentos são aplicados para o problema de estoque e roteirização (IRP) e melhoram a solução inicial em torno de 50\%.

Dror e Ball (1987) seguiram as mesmas idéias de Dror e Levy (1986). O objetivo é minimizar o custo anual de entrega e falta de estoques. O problema anual é reduzido a uma série de subproblemas semanais. Uma política ótima de reabastecimento baseada em um único cliente é desenvolvida tanto para o caso de demanda determinística quanto estocástica. A estratégia de solução é baseada na heurística de decomposição de Fischer e Jaikumar (1981) para problemas de roteirização de veículos, e consiste na determinação dos dias de atendimento dos clientes pela resolução de um problema de alocação generalizada (generalized assignment problem), seguida da determinação dos roteiros de cada dia de atendimento por meio de uma versão modificada da heurística de Clarke e Wright (1964). Após este resultado, aplica-se uma heurística de melhoria baseada na busca local.

Chien, Balakrishnan e Wong (1989) propuseram um modelo de programação inteira para resolver o problema de um único dia, baseado nas idéias de Federgruen e Zipkin (1984), descritas anteriormente para demandas estocásticas. Mas no modelo de Chien, Balakrishnan e Wong os dias não são in- dependentes, um dia influencia o outro, e as demandas são determinísticas. Passando informação de um dia para o outro, o sistema simula um modelo de planejamento de múltiplos dias. Assumindo que a taxa máxima de consumo por dia de cada cliente é conhecida, a heurística tem como objetivo maximizar o lucro final, que é o lucro por unidade entregue, menos a penalidade por unidade de demanda não satisfeita, menos o custo de entrega, em um único dia, sob a restrição que a quantidade de produtos entregues para cada cliente é no máximo sua demanda máxima. Dada a solução ótima para um dia, os resultados são utilizados para o próximo dia. $\mathrm{O}$ problema é formulado a partir de um modelo de programação inteira mista que aloca da melhor forma possível a distribuição de estoques da fábrica para os clientes, agrupa os clientes em rotas e roteiriza. Um método de relaxação lagrangiana e um método heurístico são usados para resolver o problema. O problema principal é decomposto em um subproblema de alocação de estoques e um subproblema de roteirização de veículos. Os clientes são abastecidos a partir de um único depósito. O problema de multiperíodo é decomposto em séries de subproblemas de um único período.

Herer e Levy (1997) trataram de uma versão do problema em que são considerados também os custos de manutenção de estoques e falta de produto. Os autores propuseram uma heurística temporal, que determina a melhor data de abastecimento dentro de um horizonte semanal, de forma a estabelecer uma distância temporal entre clientes. As rotas são formadas com base no método das economias proposto por Clarke e Wright (1964).

Campbell et al. (1998) e Campbell, Clarke e Savelsbergh (2002) decompõem o problema em duas etapas. Na primeira fase, é resolvido um problema de programação inteira mista que determina as quantidades a serem entregues aos clientes, os dias de atendimento e a designação de clientes por rotas. $\mathrm{Na}$ segunda fase, determina-se a programação efetiva das rotas a partir dos resultados obtidos na primeira fase. Os autores propõem um procedimento de agregação geográfica de clientes e uma agregação dos períodos de tempo à medida que se avança o horizonte de planejamento. Portanto, para o início do período de planejamento é gerada uma roteirização diária, ao passo que para o final do período gera-se uma roteirização semanal.

\section{MÉTODOS DE SOLUC̣̃̃O PARA PROBLEMAS DE ESTOQUE E ROTEIRIZAC̣ÃO COM DEMANDA ESTOCÁSTICA}

\section{Modelos agregados (modelos de freqüência de atendimento)}

Barnes-Schuster e Bassok (1997) estudaram um problema que considera demanda estocástica, no qual vários clientes são abastecidos a partir de um único depósito. Os autores consideram um sistema de entregas diretas. O estudo é ba- 
seado em simulação, e segundo os autores, o método apresenta bons resultados para políticas de entregas diretas.

Çetinkaya e Lee (2000) estudaram um problema onde o vendedor acumula pedidos durante um intervalo de tempo $T$, e após o final do intervalo de tempo são feitas as entregas aos clientes. Os autores apresentaram um modelo analítico para resolução do problema.

\section{Modelos de instante de atendimento}

Federgruen e Zipkin (1984) estudaram o problema de estoque e roteirização para um único dia e se basearam nas idéias do problema de roteirização tradicional. Há um depósito central que abastece vários clientes, com uma capacidade limitada de produto disponível, e a variável aleatória é a taxa de consumo diária de cada cliente. Para um determinado dia, o problema é alocar os estoques entre os clientes, de forma a minimizar os custos de transporte, estoque e falta no final do dia. Os custos de manutenção e falta de estoques são não-lineares. Determina-se, portanto, a cada dia, quais clientes visitar, quanto entregar para cada cliente e quais rotas seguir. O problema foi modelado como programação inteira não-linear. Devido aos custos de armazenagem e falta, e à quantidade limitada de estoque, nem todos os clientes são visitados todos os dias. Quando muito complexo, o problema pode ser decomposto em subproblemas, como um problema de alocação de estoque que determina os custos de estoque e falta, e vários problemas do caixeiro viajante, um para cada veículo, que definem os custos de transporte para cada veículo. A idéia é construir uma solução inicial e melhorar a solução alternando os clientes entre as rotas. O algoritmo termina quando não há mais melhora na solução.

O modelo de Dror e Ball (1987) descrito anteriormente também considera demanda estocástica.

Trudeau e Dror (1992) basearam-se nas idéias de Dror e Ball (1987) e contribuíram nesta linha. Um depósito central abastece os clientes. O objetivo é construir diariamente rotas de veículos que resultem em uma operação eficiente para um horizonte de longo prazo, de modo que não haja falta de produto. A eficiência da operação é medida pela média das unidades entregues durante uma hora. É importante que um horizonte de curto prazo consiga projetar o objetivo de um horizonte de longo prazo. Quando a demanda dos clientes de uma determinada rota excede a capacidade do veículo, ocorre falta de estoque e o veículo retorna ao depósito para reabastecer o restante dos clientes. Esta ocorrência é denominada "route failure".

Minkoff (1993) propôs uma heurística de decomposição para um problema que considera demanda estocástica e dinâmica. O problema é modelado como um processo de decisão de Markov. Os clientes estão distribuídos geograficamente e são abastecidos através de um depósito central.

Bassok e Ernst (1995) consideram um problema de alocação dinâmica de múltiplos produtos a vários clientes, a partir de um depósito central. A cada instante em que o distribuidor visita um determinado cliente, ele tem a informação a respeito da demanda deste cliente, mas ainda não conhece as demandas dos demais clientes. O modelo determina quanto entregar de mercadoria para cada cliente, considerando diferentes penalidades de falta de produto para cada cliente.

Bard et al. (1998) estudaram um modelo de decomposição para o problema de estoque e roteirização com "satellite facilities". "Satellite facilities" são outros locais, além do depósito central, onde os veículos podem ser reabastecidos e continuar a entrega de mercadorias. É apresentada uma metodologia que permite decompor um problema de longo prazo, e assim resolvê-lo diariamente. Determina-se a freqüência ótima de reabastecimento para cada cliente, similar às idéias de Dror e Ball (1987), para um horizonte de tempo de duas semanas. Os clientes são abastecidos a partir de um depósito central e de "satellite facilities", e o fornecimento de produtos é ilimitado. A única diferença entre o depósito e "satellite facilitie" é que o depósito é o ponto de origem e destino de cada veículo. Os veículos saem do depósito, visitam vários clientes, reabastecem em um dos "satellite facilities" se necessário, atendem outro subgrupo de clientes, reabastecem novamente em um dos "satellite facilities", e no final da rota retornam ao depósito. As entregas em um determinado dia devem ser completadas dentro de um intervalo de $T$ horas. Três heurísticas são desenvolvidas para resolver o problema de roteirização de veículos com "satellite facilities". O objetivo é minimizar os custos de entregas anuais, de modo que não haja falta de estoques em nenhum momento. Os veículos retornam ao depósito central no final do dia. O desempenho é medido através de dois parâmetros: a distância percorrida e o custo incremental total.

Reiman, Rubio e Wein (1999) estudaram um problema de estoque e roteirização com demanda estocástica, onde um único veículo abastece diversos clientes. O objetivo é minimizar os custos totais de estoque (manutenção e falta) e os custos de transporte. Os autores propõem uma estratégia de decomposição baseada na escala de tempo.

Kleywegt, Nori e Savelsbergh (2002) estudaram um problema de estoque e roteirização com entregas diretas. O problema foi formulado por um processo de decisão de Markov, e os autores propuseram algoritmos aproximados para solução do modelo. Os clientes são reabastecidos a partir de um depósito central, por uma frota de veículos homogênea.

Kleywegt, Nori e Savelsbergh (2004) estudaram um problema de estoque e roteirização com múltiplas entregas. Da mesma forma que em Kleywegt, Nori e Savelsbergh (2002), desenvolve-se um modelo markoviano de decisão, que é resolvido por programação dinâmica, através de métodos de aproximação.

O Quadro 2 apresenta uma síntese dos métodos apresentados, de acordo com a classificação de Baita et al. (1998). 


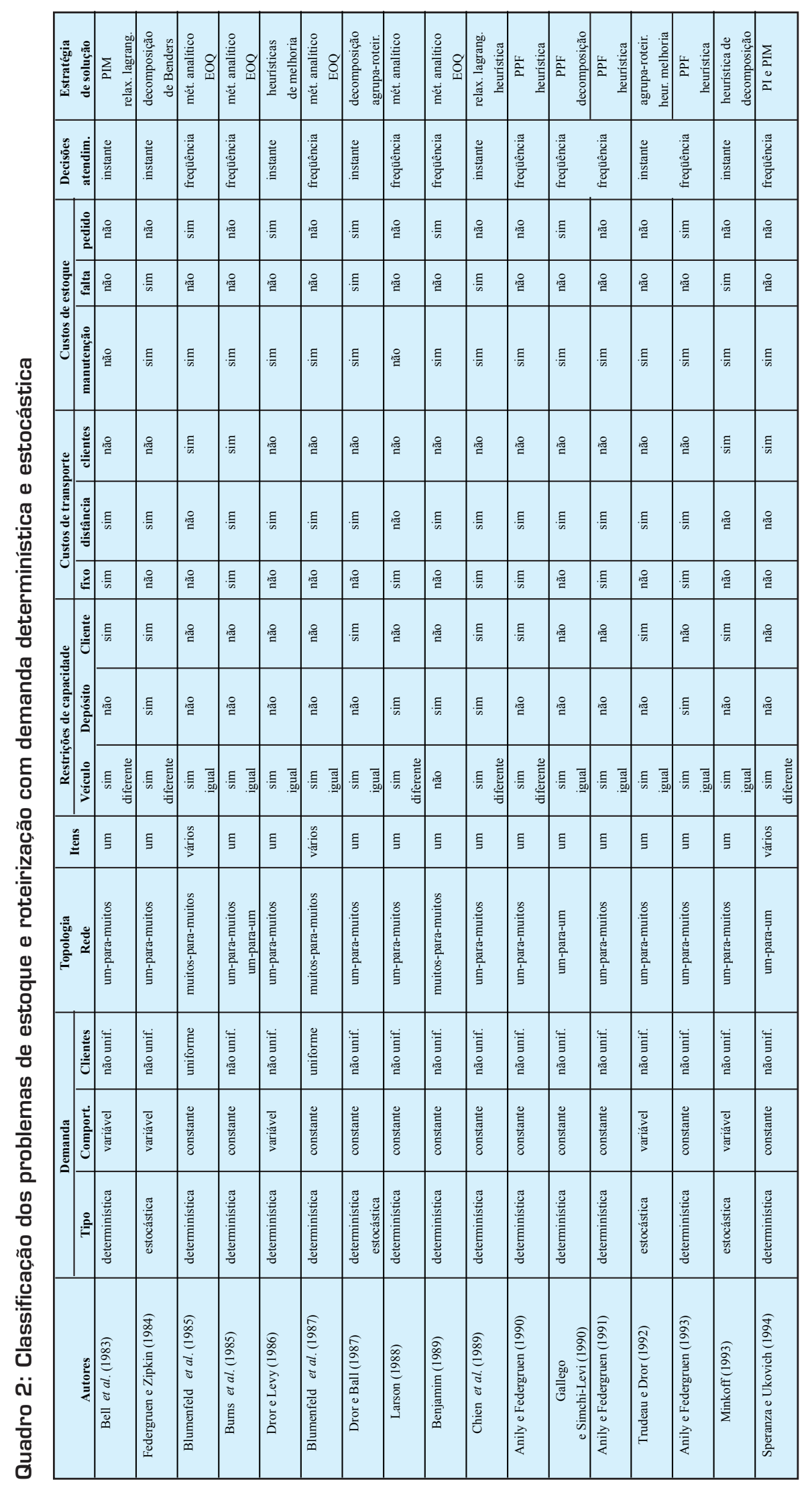




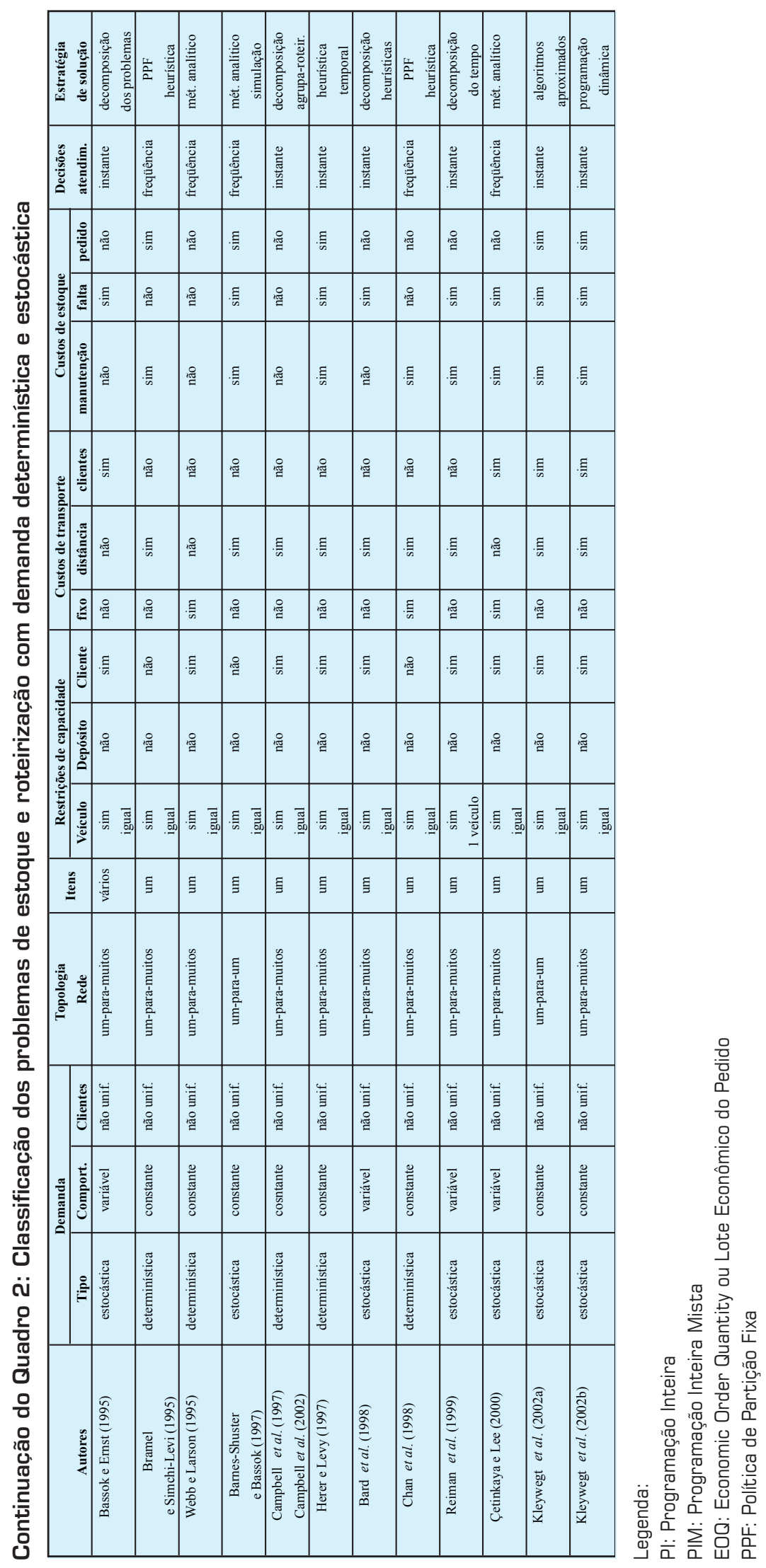




\section{CONSIDERAC̣ÕES FINAIS}

O objetivo deste trabalho foi estudar os principais métodos de solução encontrados na literatura para resolver o problema de estoque e roteirização, tanto para demanda determinística quanto estocástica.

Os modelos estocásticos, em geral, requerem uma grande quantidade de dados históricos e um tratamento estatístico adequado, que não é uma tarefa simples. Desta forma, apesar dos problemas de estoque e roteirização serem de natureza estocástica, a maioria dos problemas encontrados na literatura é de natureza determinística.
Considerando a bibliografia analisada, podemos concluir também que os modelos de estoque e roteirização buscam conciliar duas funções conflitantes, tradicionalmente tratadas de maneira independente pelas empresas, o que contribui para a integração e otimização da cadeia de distribuição como um todo.

A implementação de metaheurísticas (busca tabu, simulated annealing, algoritmos genéticos) tem sido bastante utilizada para resolução de problemas de grande escala, como problemas de produção e roteirização de veículos. Com base nos modelos analisados neste trabalho, conclui-se que nenhuma metaheurística foi implementada em problemas de estoque e roteirização.

\section{Artigo recebido em 04/03/2005 Aprovado para publicação em 16/05/2006}

\section{n Referências Bibliográficas}

ANILY, S.; FEDERGRUEN, A. One Warehouse Multiple Retailer Systems with Vehicle Routing Costs. Management Science, v. 36, n. 1, p. 92-114, 1990

ANILY, S.; FEDERGRUEN, A. Rejoinder to Comments on One-Warehouse Multiple Retailer Systems with Vehicle Routing Costs. Management Science, v. 37, n. 11, p. $1497-1499,1991$

ANILY, S.; FEDERGRUEN, A. Two-Echelon Distribution Systems with Vehicle Routing Costs and Central Inventories. Operations Research, v. 41, n. 1, p. $37-$ 47, 1993.

BAITA, F.; UKOVICH, W.; PESENTI, R. e FAVARETTO, D. Dynamic Routingand-Inventory Problems: A Review. Transportation Research A, v. 32, n. 8, p. 585-598, 1998.

BARD, J. F.; HUANG, L.; JAILLET, P. e DROR, M. A Decomposition Approach to the Inventory Routing Problem with Satellite Facilities. Transportation Science, v. 32, n. 2, p.189-203, 1998

BARNES-SCHUSTER, D.; BASSOK, Y Direct Shipping and the Dynamic Singledepot/Multi-retailer Inventory System. European Journal of Operational Research, v. 101, n. 3, p. 509-518, 1997.
BASSOK, Y.; ERNST, R. Dynamic Allocations for Multi-Product Distribution. Transportation Science, v. 29, n. 3, p. 256-266, 1995.

BELL, W.; DALBERTO, L.; FISHER, M.; GREENFIELD, A.; JAIKUMAR, R.; KEDIA, P.; MACK, R.; PRUTZMAN, P. Improving the Distribution of Industrial Gases with an On-Line Computerized Routing and Scheduling Optimizer. Interfaces, v. 13 , n. 6 , p. $4-23,1983$

BENJAMIN, J. An Analysis of Inventory and Transportation Costs in a Constrained Network. Transportation Science, v. 23, n. 3, p. 177-183, 1989.

BLUMENFELD, D. E.; BURNS, L. D.; DILTZ, J. D.; DAGANZO, C. F. Analyzing Trade-Offs between Transportation, Inventory and Production Costs on Freight Networks. Transportation Research $B$, v. 19 B, n. 5, p. $361-380,1985$.

BLUMENFELD, D.E.; BURNS, L. D. DAGANZO, C.F.; FRICK, M. C.; HALL, R. W. Reducing Logistics Costs at General Motors. Interfaces, v. 17, n. 1, p. 26-47, 1987.

BRAMEL, J: SIMCHI-LEVI, D. A Location Based Heuristic for General Routing Problems. Operations Research, v. 43, n. 4, p. 649-660, 1995
BURNS, L. D. HALL, R. W: BLUMENFELD, D. E. DAGANZO, C. F. Distribution Strategies that Minimize Transportation and Inventory Costs. Operations Research, v. 33, n. 3, p. 469-490, 1985.

CAMPBELL, A.; CLARKE, L.; KLEYWEGT, A.; SAVELSBERGH, M. W. P. The Inventory Routing Problem. In: Fleet Management and Logistics, GRAINIC, T. G., LAPORTE, G. (eds), Kluwer Academic Publishers, p. 95-113, 1998.

CAMPBELL, A.; CLARKE, L.; SAVELSBERGH, M.W.P. Inventory Routing in Practice. In: The Vehicle Routing Problem, TOTH, P., VIGO, D. (eds), SIAM monographs on discrete mathematics and applications, p. 309-330, 2002.

CETINKAYA, S. LEE, C. Y Stock Replenishment and Shipment Scheduling for Vendor Managed Inventory Systems. Management Science, v. 46, n. 2, p. 217 232, 2000.

CHAN, L. M. A.; FEDERGRUEN, A.; SIMCHI-LEVI, D. Probabilistic Analysis and Practical Algorithms for InventoryRouting Models. Operations Research, v. 46, n. 1, p. 96-106, 1998.
CHIEN, T. W.; BALAKRISHNAN, A.; WONG; R. T. An Integrated Inventory Allocation and Vehicle Routing Problem. Transportation Science, v. 23, n. 2, p. 67-76, 1989.

CLARKE, G.; WRIGHT, J. W. Scheduling of Vehicles from a Central Depot to a Number of Delivery Points. Operation Research, v. 12, n. 4, p. 568-581, 1964.

DROR, M.; BALL, M. Inventory/Routing: Reduction from an Annual to a Short Period Problem. Naval Research Logistics Quarterly, v. 34, n. 6, p. 891-905, 1987.

DROR, M.; LEVY, L. A Vehicle Routing Improvement Algorithm Comparison of a "Greedy" and a Matching Implementation for Inventory Routing. Computers and Operations Research, v. 13, n. 1, p. 3345, 1986.

FEDERGRUEN, A.; ZIPKIN, P. A Combined Vehicle Routing and Inventory Allocation Problem. Operations Research, v. 32, n. 5 , p. 1019-1037, 1984.

FISHER, M.; JAIKUMAR, R. A Generalized Assignment Heuristic for Vehicle Routing Networks, v. 11, n. 2, p. 109-124, 1981. 


\section{- Referências Bibliográficas}

GALLEGO, G. SIMCHI-LEVI, D. On the Effectiveness of Direct Shipping Strategy for the One-Warehouse Multi-Retailer R-Systems. Management Science, v. 36, n. 2, p. $240-243,1990$

HALL, R. W. Comments on One-warehouse multiple retailer systems with vehicle routing costs. Management Science, v. 37, n. 11 , p. 1496-1497, 1991.

HERER, Y. T.; LEVY, R. The metered inventory routing problem, an integrative heuristic algorithm. International Journal of Production Economics, v. 51, n. 1-2, p. 69-81, 1997.

HOLMSTRÖM, J. Implementing VendorManaged Inventory the Efficient Way: a case study of partnership in the Supply Chain. Production and Inventory Management Journal, v. 39, n. 3, p. 1-5, 1998.
KLEYWEGT, A. J· NORI, V.S. Savelsbergh, W.P. The Stochastic Inventory Routing Problem with Direct Deliveries. Transportation Science, v. 36, n. 1, p. 94-118, 2002.

KLEYWEGT, A. J.; NORI, V. S. SAVELSBERGH, W. P. Dynamic Programming Approximations for a Stochastic Inventory Routing Problem. Transportation Science, v.38, n.1, p. 4270, 2004.

KLINGENBERG, C. O.; ANTUNES, J. A V. J. Construção de um modelo orientativo para a implantação do VMI em empresas da indústria supermercadista brasileira. In: XXXVII Assembléia do Conselho Latino-Americano de Escolas de Administração, 2002.

LARSON, R. C. Transporting Sludge to the 106-Mile Site: An Inventory/Routing
Model for Fleet Sizing and Logistics System Design. Transportation Science, v. 22, n. 3, p. 186-198, 1988.

LEE, H. L.; PADMANABHAN, V.; WHANG, S. Information distortion in a supply chain: the bullwhip effect. Management Science, v. 43, n. 4, p. 546-558, 1997.

MINKOFF, A. S. A Markov Decision Model and Decomposition Heuristic for Dynamic Vehicle Dispatching. Operations Research, v. 41, n. 1, p. 77-60, 1993.

REIMAN, M. I.; RUBIO, R.; WEIN, L. M. Heavy Traffic Analysis of the Dynamic Stochastic Inventory-Routing Problem. Transportation Science, v. 33, n. 4, p. 361-380, 1999.

SPERANZA, M. G.; UKOVICH, W. Minimizing Transportation and Inventory
Costs for Several Products on a Single Link. Operations Research, v. 42, n. 5, p. 879-894, 1994

TRUDEAU, P.; DROR, M. Stochastic Inventory Routing: Route Design with Stockouts and Route Failures. Transportation Science, v. 26, n. 3, p. 171-184, 1992

WEBB, R.; LARSON, R. Period and Phase of Customer Replenishment: A New Approach to the Strategic Inventory/ Routing Problem. European Journal of Operational Research, v. 85, n. 1, p. 132 148,1995

ZNAMENKY, A.; CUNHA, C. B. O problema de estoque-roteirização com demanda determinística. Revista Transportes, v. XI, p. 31-40, dezembro 2003 .

2o Congresso ECR Brasil. O ECR no BrasilScorecard, São Paulo, 2000.

\section{- Sobre o autor}

\section{Patrícia Prado Belfiore}

Doutora em Engenharia de Produção - POLI-USP

Professora do Departamento de Engenharia de Produção do Centro Universitário da FEI

Endereço: Av. Humberto A. C. Branco, 3972 - Assunção - São Bernardo do Campo - Brasil

CEP: 09850-901

Tel. / Fax: (55) (11) 4353-2900

E-mail: patricia.belfiore@labfin.com.br

\section{Oswaldo Luiz do Valle Costa}

Professor Titular do Departamento de Engenharia de Telecomunicações e Controle - POLI-USP

Endereço: Av. Prof. Luciano Gualberto, trav.3, 158 - Sala C2-03 - Cidade Universitária - São Paulo - SP

CEP: 05508-900

Tel. / Fax: (55) (11) 3091-5771

E-mail: oswaldo@lac.usp.br

\section{Luiz Paulo Lopes Fávero}

Professor Doutor do Departamento de Contabilidade e Atuária - FEA-USP

Endereço: Av. Prof. Luciano Gualberto, 908 - FEA 3 - Sala 237 - Cidade Universitária - São Paulo - Brasil.

CEP: 05508-900

Tel. / Fax: (55) (11) 3091-5820

E-mail: lpfavero@usp.br 